\title{
Presencia de Porphyromonas gingivalis, Tannerella forsythia, Treponema denticola y Aggregatibacter actinomycetemcomitans en el biofilm subgingival de pacientes diabéticos tipo 2: estudio transversal
}

\author{
Presence of Porphyromonas gingivalis, Tannerella forsythia, Treponema denticola \\ and Aggregatibacter actinomycetemcomitans in the subgingival biofilm of diabetic \\ mellitus 2 patients: a cross sectional study
}

Quintero $\mathrm{AJ}^{1}$, Prada $\mathrm{P}^{2}$, Inostroza $\mathrm{CM}^{3}$, Chaparro $\mathrm{A}^{1}$, Sanz $\mathrm{AF}^{1}$, Ramírez $\mathrm{VL}^{4}$, Morales $\mathrm{HC}^{5}$

\begin{abstract}
RESUMEN
Antecedentes: La investigación de la microflora subgingival en pacientes diabéticos tipo 2 con periodontitis ha presentado resultados contradictorios. Objetivo: Determinar la presencia de Porphyromonas gingivalis, Tannerella forshytia, Treponema denticola y Aggregatibacter actinomycetemcomitans, en el biofilm subgingival de pacientes diabéticos tipo 2 y relacionarlo con el grado de control metabólico. Método: Estudio descriptivo transversal, en el cual se analizaron 23 pacientes diabéticos derivados consecutivamente del Policlínico de Especialidades de la Universidad de los Andes. Previo consentimiento informado, se realizó un examen clínico periodontal que incluyó mediciones de profundidad al sondaje, nivel de inserción clínica y sangrado gingival. Fueron clasificados según severidad de periodontitis y control metabólico de la diabetes determinado por un promedio de 3 exámenes de hemoglobina glicosilada. La detección microbiológica se realizó mediante la técnica de reacción en cadena de la polimerasa. Resultados: En el grupo de pacientes estudiados, Treponema denticola y Tannerella forsythia fueron las bacterias más prevalentes (65.2\%), seguida por Porphyromonas gingivalis (17.3\%) y Aggregatibacter actinomycetemcomitans (13\%). Los pacientes con peor control glicémico tuvieron una mayor presencia de Treponema denticola, Tannerella forsythia, Porphyromonas gingivalis y Agreggatibacter actinomycetemcomitans y un aumento en el índice de sangrado al sondaje. Conclusiones: En el grupo de pacientes diabéticos estudiado, las bacterias más prevalentes fueron Treponema denticola y Tannerella forsythia. Los pacientes diabéticos tipo 2 con moderado y mal control glicémico presentaron mayor presencia de los microorganismos estudiados, comparado con los grupos con mejores niveles de control glicémico.
\end{abstract}

Rev. Clin. Periodoncia Implantol. Rehabil. Oral Vol. 4(2); 54-58, 2011.

Palabras clave: Diabetes mellitus, patógenos periodontales, control glicémico.

\section{ABSTRACT}

Background: The investigation of subgingival microflora in type 2 diabetic patients with periodontitis presented conflicting results. Aim: To determine the presence of Porphyromonas gingivalis, Tannerella forshytia, Treponema denticola and Aggregatibacter actinomycetemcomitans in subgingival biofilm of patients with diabetes type 2 and to relate it to the degree of metabolic control. Method: A descriptive study, which analyzed 23 diabetic patients consecutively referred from the Internal Medicine Unit of Medicine Faculty at Universidad de los Andes was conducted. After obtaining an informed consent from the patients a clinical examination that included measurements of periodontal pocket depth, clinical attachment level and gingival bleeding was performed. The patients were classified according to the severity of periodontitis and metabolic control of diabetes as determined by an average of 3 of glycosylated haemoglobin tests. Microbial technique was performed by chain reaction of polymerase. Results: In the group of patients examined the most prevalent bacteria were, Treponema denticola and Tannerella forsythia (65.2\%), followed by Porphyromonas gingivalis (17.3\%) and Aggregatibacter actinomycetemcomitans (13\%). Patients with poor glycemic control had a greater presence of Treponema denticola, Tannerella forsythia, Porphyromonas gingivalis and Agreggatibacter actinomycetemcomitans and an increase in the rate of bleeding on probing. Conclusions: In the group of diabetic patients studied, the most prevalent bacteria were Treponema denticola and Tannerella forsythia. Type 2 diabetic patients with moderate and poor glycemic control had a higher presence of these microorganisms, compared to groups with higher levels of glycemic control.

Rev. Clin. Periodoncia Implantol. Rehabil. Oral Vol. 4(2); 54-58, 2011.

Key words: Diabetes mellitus, periodontal pathogens, and glycemic control.

INTRODUCCIÓN

La Diabetes Mellitus (DM) constituye un grupo heterogéneo de desórdenes que afectan el metabolismo de carbohidratos, lípidos y proteínas ${ }^{(1)}$. Se caracteriza por presentar hiperglicemia, que a largo plazo afecta distintos tejidos como el corazón, ojos, riñones, nervios y el sistema vascular ${ }^{(2)}$. Según la última encuesta realizada a nivel nacional, en el año 2009, por el Ministerio de Salud, la prevalencia es de $9.4 \%$ de la población ${ }^{(3)}$.

Estudios epidemiológicos han mostrado una mayor prevalencia y severidad de periodontitis en pacientes diabéticos comparados con pacientes no diabéticos ${ }^{(4,5)}$. También se ha señalado que formas severas de periodontitis pueden tener un impacto significativo en el control metabólico del paciente diabético6, incrementando la resistencia a la insulina y empeorando el estado metabólico y aumentando sus complicaciones ${ }^{(7,8)}$.

1. Profesor Asistente. Departamento de Periodoncia. Facultad de Odontología, Universidad de los Andes. Chile.

2. Cirujano Dentista. Universidad de los Andes. Chile.

3. Bioquímico. Magíster en Ciencias Biológicas. Centro de Biología y Regeneración Oral (CIBRO). Facultad de Odontología, Universidad de los Andes. Chile.

4. Magíster Salud Pública. Departamento de Ciencias, Investigación y Gestión. Facultad de Odontología, Universidad de los Andes. Chile.

5. Jefe Departamento de Medicina Interna. Facultad de Medicina, Universidad de los Andes. Chile. 
La microbiología de la periodontitis en pacientes con diabetes tipo 1 ha sido reportada en varios estudios, sin embargo no existe suficiente información en la Diabetes Mellitus $2^{(9-11)}$. Según algunos estudios los pacientes diabéticos tiene una proporción similar de periodontopatógenos a la de los no diabéticos ${ }^{(10,11)}$, mientras que otros ensayos indican una mayor prevalencia de patógenos periodontales como Porphyromonas gingivalis, Prevotella intermedia, Campylobacter rectus y Capnocytophaga en pacientes diabéticos comparados con no diabéticos ${ }^{(10,12)}$. Ciertamente la microflora subgingival de pacientes diabéticos con enfermedad periodontal es pobremente entendida y los resultados contradictorios ${ }^{(9)}$. El objetivo de este estudio es determinar la presencia de Porphyromonas gingivalis (Pg), Tannerella forshytia (Tf), Treponema denticola (Td) y Aggregatibacter actinomycetemcomitans (Aa) en el biofilm subgingival de pacientes diabéticos tipo 2 .

\section{MÉTODO}

\section{Selección de Pacientes}

Se desarrolló un estudio descriptivo transversal donde se incluyeron pacientes mayores de 30 años, de ambos sexos, en tratamiento por diabetes mellitus tipo 2 (DM 2) $)^{(13)}$ que fueron seleccionados de forma consecutiva y derivados durante los meses de Mayo a Septiembre del año 2010 desde el Policlínico de Diabetes del Centro de Especialidades Médicas de la Universidad de los Andes en San Bernardo, con mediciones de hemoglobina glicosilada (HbA1c) de sus últimos tres controles médicos y con al menos 12 dientes en boca. Se excluyeron aquellos pacientes con enfermedades sistémicas como insuficiencia renal, insuficiencia respiratoria crónica, artritis reumatoide, enfermedad coronaria, presencia de otras infecciones, en tratamiento con antibióticos en los últimos 6 meses y/o con antecedentes de haber recibido tratamiento periodontal. Este protocolo fue aprobado por el comité de ética de la Facultad de Odontología de la Universidad de los Andes. Previo al estudio todos los pacientes consintieron voluntariamente en su participación. Todos los pacientes recibieron tratamiento periodontal.

\section{Determinación del Estado Periodontal}

Un examinador, previamente calibrado, quién desconocía el nivel de control glicémico del paciente, realizó el examen periodontal clínico. Se examinaron todas las piezas dentarias presentes en boca exceptuando los terceros molares. Utilizando una sonda periodontal No 15 de la U. de Carolina del Norte se midió profundidad al sondaje (PS) y nivel de inserción clínico (NIC) en 6 sitios por cada diente. Además se determinó el índice de sangrado al sondaje (IS) observando las superficies dentarias sangrantes, después de 20 segundos de realizado un recorrido del margen gingival con la sonda periodontal, y promediándolo con las superficies totales examinadas.

\section{Toma de Muestra de Bacterias Subgingivales}

La muestra subgingival fue recolectada desde los dos sacos periodontales más profundos de cada cuadrante. Los sitios seleccionados fueron aislados con torundas de algodón, libres de saliva o sangre, y la placa supragingival evidente fue removida cuidadosamente con cureta. Posteriormente se introdujo en el saco seleccionado un cono de papel estéril número 40, por 20 segundos. Los conos fueron almacenados secos en tubos Eppendorf estériles, rotulados y congelados a $-20^{\circ} \mathrm{C}$ hasta su análisis, el que se realizó entre 1 y 3 semanas posteriores a la toma de la muestra.

Determinación de Patógenos Mediante Técnica de Reacción en Cadena de la Polimerasa (PCR) Convencional

Para la purificación del ADN bacteriano, se utilizó un cono, seco y estéril, por cada muestra, el cual fue transportado a un tubo Eppendorf estéril con $120 \mu \mathrm{L}$ de agua libre de nucleasas. En la máquina de PCR se realizó un ciclo de denaturación a $94^{\circ} \mathrm{C}$ por 10 minutos. Posteriormente se centrifugó a alta velocidad por 10 segundos, el DNA templado $(100 \mu \mathrm{L})$ se traspasó a un tubo Eppendorf estéril y se almacenó a $-20^{\circ} \mathrm{C}$, desechando el cono de la muestra.

Se utilizó la técnica de PCR convencional para determinar la presencia de $\mathrm{Pg}, \mathrm{Tf}, \mathrm{Td}$ y Aa. Para la reacción de PCR se utilizó $9.2 \mu \mathrm{L}$ de DNA templado + $\mathrm{H} 20$ más $10.8 \mu \mathrm{L}$ de PCR Master Mix; que contiene 10 $\mu \mathrm{L}$ de Go Taq Green Master Mix (Promega, USA), $0.4 \mu \mathrm{L}$ de primer 1 (25 $\mu \mathrm{M})$ y $0.4 \mu \mathrm{L}$ de primer $2(25 \mu \mathrm{M})$. El programa de PCR se realizó con un ciclo de denaturación por 5 minutos a $94^{\circ} \mathrm{C}$ y 40 ciclos de denaturación a $94^{\circ} \mathrm{C}$ por 30 segundos, apareamiento de partidores a $55^{\circ} \mathrm{C}$ por 30 segundos y elongación a $72^{\circ} \mathrm{C}$ por 45 segundos. La elongación final fue a $72^{\circ} \mathrm{C}$ por 10 minutos. Las muestras fueron conservadas a $-20^{\circ} \mathrm{C}$. Los productos finales del PCR se visualizaron en un gel de agarosa al $1.5 \%$. (Figura 1) y fueron visualizados con tinción SybrSafe (Invitrogen) en un transiluminador de luz ultravioleta (Invitrogen). Los partidores de PCR utilizados en la determinación de las especies bacterianas analizadas se observan en la Tabla 1

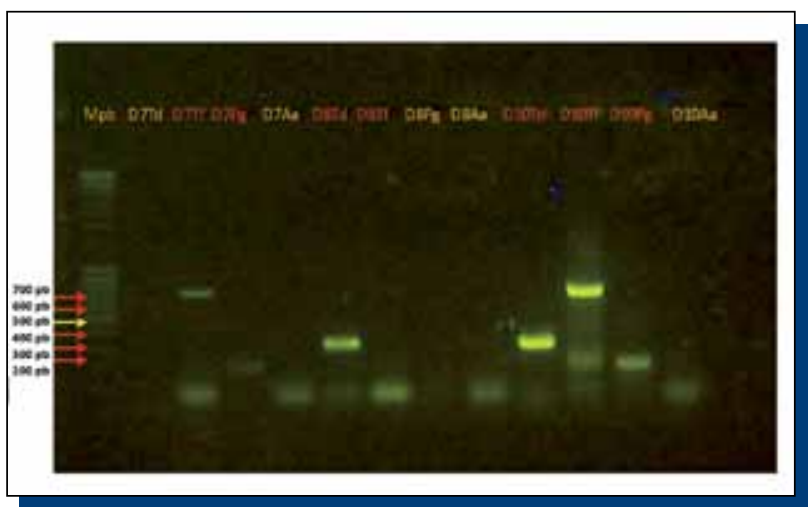

Figura 1. Resultados PCR en gel de agarosa 1.5\%. Mpb: Marcador molecular; Td: Treponema denticola; Tf: Tannerella Forsythia; Pg: Porphyromonas gongivalis; Aa: Aggregatibacter actinomycemtencomitnas.

Tabla 1. Partidores utilizados en el análisis con PCR.

\begin{tabular}{|c|c|c|c|}
\hline Nombre & Bacteria & Secuencia & Tamaño \\
\hline Td-1 & T. $d$ & $5{ }^{\circ}$ taa tac cga atg tgc tca ttt aca $t-3^{\prime}$ & $316 \mathrm{pb}$ \\
\hline Td-2 & & $5^{\circ}-$ tac aag aag cat tcc ctc ttc ttc tta- $3^{`}$ & \\
\hline Tf-1 & T.f & $5{ }^{\circ}-$ tac agg gga ata aaa tga gat acg-3 & $745 \mathrm{pb}$ \\
\hline Tf-2 & & $5^{\prime}$-acg tca tcc cca cct tcc tc-3' & \\
\hline $\mathrm{Pg}-1$ & P. $g$ & $5^{\prime}$-tgt aga tga ctg aaa acc-3' & $197 \mathrm{pb}$ \\
\hline $\mathrm{Pg}-2$ & & $5^{\prime}$-acg tca tcc cca cct tcc tc- $3^{\prime}$ & \\
\hline Aa-1 & A. $a$ & $5^{\prime}$-attg ggg ttt agc cct ggt g-3' & $360 \mathrm{pb}$ \\
\hline Aa-2 & & $5^{\prime}$-acg tca tcc cca cct tcc tc- $3^{\prime}$ & \\
\hline
\end{tabular}

\section{Determinación del Estado Metabólico}

El estado metabólico de los pacientes fue determinado mediante la media de las mediciones de los 3 últimos controles de hemoglobina glicosilada ( $\mathrm{HbAc} 1)$. De acuerdo a esto, fueron divididos en pacientes con buen control glicémico si su media era inferior o igual a $6,9 \%$; moderado control si estaba entre 7.0 y $7.9 \%$ y mal control si su promedio era de $8 \%$ o más.

\section{Determinación del Diagnóstico Periodontal}

Los pacientes fueron divididos de acuerdo a la severidad de periodontitis presente en leves, moderados y severos. Para esto se les clasificó según se indica en la Tabla 2.

Tabla 2. Determinación de severidad de enfermedad periodontal ${ }^{(14)}$.

\begin{tabular}{|l|l|l|l|}
\hline $\begin{array}{l}\text { Clasificación de } \\
\text { Periodontitis }\end{array}$ & N.I.C. & P.S. \\
\hline Periodontitis Severa & $\begin{array}{l}\geq 2 \text { sitios interproximales } \\
\text { con NIC =6 mm } \\
\text { (no en el mismo diente) }\end{array}$ & Y & $\begin{array}{l}\geq 1 \text { sitio } \\
\text { interproximal con } \\
\text { PS =5 mm }\end{array}$ \\
\hline Periodontitis Moderada & $\begin{array}{l}\geq 2 \text { sitios interproximales } \\
\text { con NIC =4 mm } \\
\text { (no en el mismo diente) }\end{array}$ & o & $\begin{array}{l}\geq 2 \text { sitios } \\
\text { interproximales } \\
\text { con PS =5 mm } \\
\text { (no en el mismo } \\
\text { diente) }\end{array}$ \\
\hline Periodontitis Leve & $\begin{array}{l}\text { No califica para Periodontitis } \\
\text { moderada o severa }\end{array}$ & & \\
\hline
\end{tabular}




\section{Análisis Estadístico}

Se describieron las variables continuas (edad, PS, NIC, IS) con medidas de tendencia central, dispersión y posición. Las variables cualitativas (tipo de bacteria, severidad de diabetes, nivel de periodontitis, sexo) se tabularon, presentándose con frecuencias absolutas y porcentuales. Las variables fueron analizadas en el programa estadístico STATA 10.

\section{RESULTADOS}

Se analizaron 23 pacientes, 8 hombres y 15 mujeres, con una media de edad de 55 años, con una desviación estándar de 11.5 años. La edad media de los sujetos con buen, moderado y mal control glicémico fue de 45, 61 y 53 años respectivamente. La media de hemoglobina glicosilada fue de $6.3 \%, 7.2 \%$ y $9 \%$ respectivamente para cada grupo. Tres de los sujetos eran fumadores y se distribuyen en los grupos de buen y moderado control glicémico (Tabla 3 ).

Tabla 3. Resumen datos demográficos y metabólicos de los pacientes con Diabetes mellitus tipo 2.

\begin{tabular}{|l|l|l|l|}
\hline \hline Variable & $\begin{array}{l}\text { Buen } \\
\text { Control } \\
\text { Glicémico } \\
\mathbf{( N = 5 )}\end{array}$ & $\begin{array}{l}\text { Moderado Control } \\
\text { Glicémico } \\
(\mathbf{N}=11)\end{array}$ & $\begin{array}{l}\text { Mal Control } \\
\text { Glicémico } \\
(\mathbf{N}=7)\end{array}$ \\
\hline $\begin{array}{l}\text { Edad media en } \\
\text { años } \\
\text { (min-max) }\end{array}$ & $44.6(31-60)$ & $60.7(52-82)$ & $53.0(32-63)$ \\
\hline Femenino & 4 & 6 & 5 \\
\hline Masculino & 1 & 5 & 2 \\
\hline $\begin{array}{l}\text { \% medio de } \\
\text { Hb1Ac } \\
\text { (\% min-max) }\end{array}$ & $6.3(5.8-6.8)$ & $7.2(7.0-8.0)$ & $9.0(8.3-10.0)$ \\
\hline $\begin{array}{l}\text { Duración DM2 } \\
\text { (años) }\end{array}$ & $6(2-15)$ & $11.18(4-25)$ & $7.57(1-17)$ \\
\hline Tabaco & 1 & 2 & 0 \\
\hline
\end{tabular}

Hb1Ac: Hemoglobina glicosilada; DM2: Diabetes mellitus tipo 2.

$21.74 \%$ de los sujetos examinados fueron diagnosticados con periodontitis leve, un $13,04 \%$ con periodontitis moderada y un $65.22 \%$ con periodontitis severa (Figura 2).

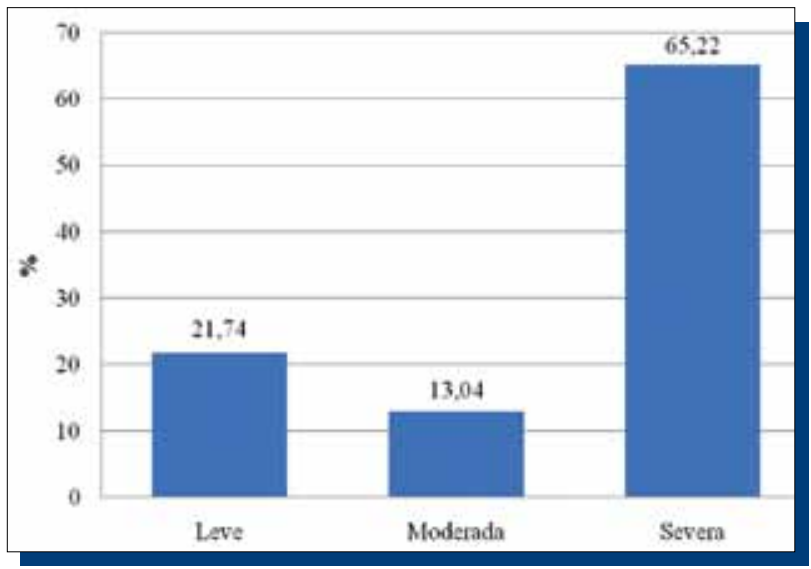

Figura 2. Distribución porcentual de la severidad de periodontitis en los sujetos con DM2.

Se evalúo la situación clínica periodontal según el nivel de control glicémico de los pacientes (Tabla 4). En la población estudiada, la profundidad al sondaje promedio fue de 2.30 (ds: 0.03), 2.77 (ds: 0.49) y 2.79 (ds: 0.73) para los grupos de buen, moderado y mal control glicémico respectivamente. El nivel de inserción clínica promedio para los mismos grupos de pacientes fue de 2.86 (ds: 0.46 ), 4.03 (ds: 1.25), 3.52 (ds: 1.18). El índice de sangrado al sondaje aumentó a medida que empeoró el control glicémico (Figura 3). En relación al número de dientes en boca este disminuye a medida que empeora el control glicémico (Figura 4).
Tabla 4. Descripción de estado clínico periodontal de los pacientes con DM 2.

\begin{tabular}{|l|l|l|l|}
\hline Variable & $\begin{array}{l}\text { Buen Control } \\
\text { Glicémico } \\
\text { (N=5) }\end{array}$ & $\begin{array}{l}\text { Moderado Contro } \\
\text { Glicémico } \\
\text { (N=11) }\end{array}$ & $\begin{array}{l}\text { Mal Control } \\
\text { Glicémico } \\
\text { (N=7) }\end{array}$ \\
\hline Mediana dientes & 22 & 21 & 14 \\
\hline PS (media y ds) & $2.30 \mathrm{ds:} 0.03$ & 2.77 ds: 0.49 & 2.79 ds: 0.73 \\
\hline NIC (media y ds) & 2.86 ds: 0.46 & 4.03 ds: 1.25 & 3.52 ds: 1.18 \\
\hline IS (media y ds) & 63.56 ds: 27.37 & 68.37 ds: 29.79 & 84.17 ds: 22.19 \\
\hline
\end{tabular}

PS: Profundidad al sondaje; NIC: Nivel de inserción clínica; IS: Índice de sangramiento.

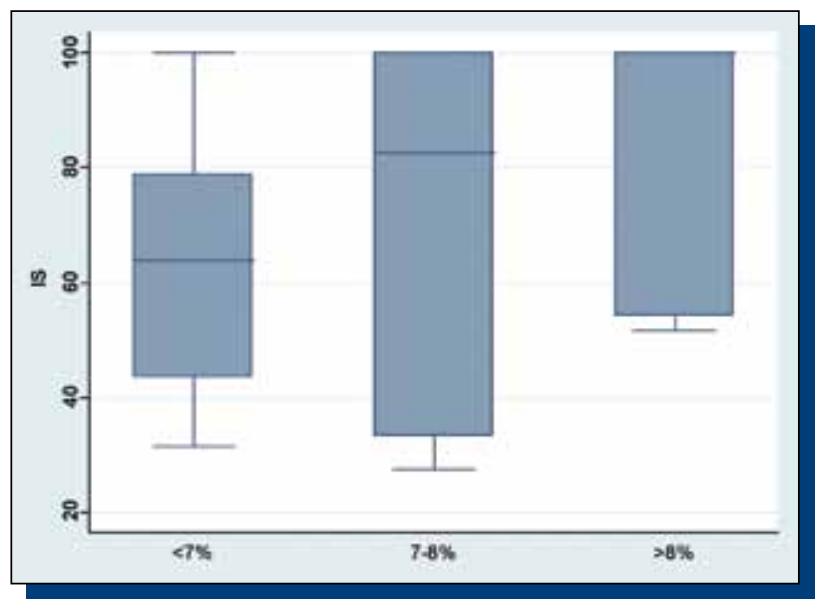

Figura 3. Índice de sangrado según nivel de control glicémico.

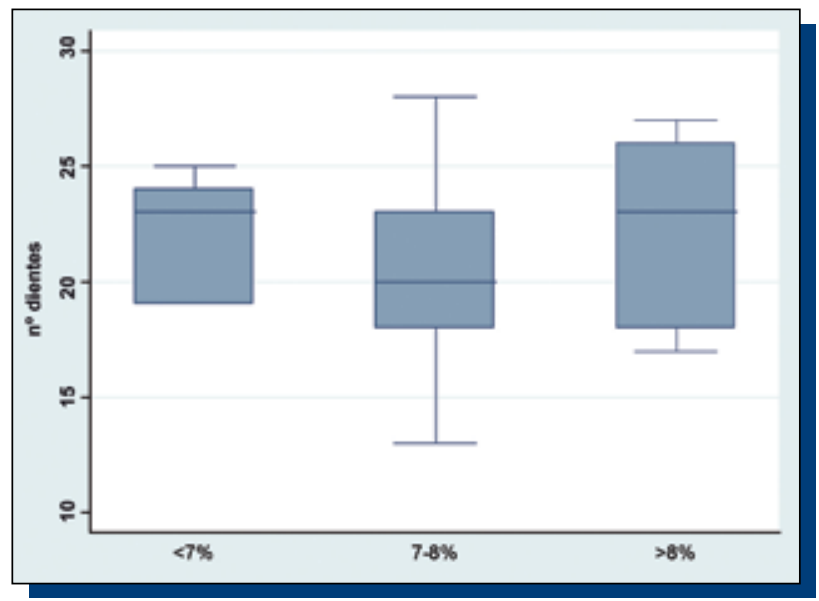

Figura 4. Número de dientes según nivel de control glicémico.

La frecuencia de detección de los patógenos periodontales se aprecia en la Tabla 5. Treponema denticola y Tannerella forsythia fueron las bacterias más prevalentes, en el $65.2 \%$ de los pacientes, seguida por Porphyromonas gingivalis (17.3\%) y Aggregatibacter actinomycetemcomitans (13\%).

Tabla 5. Especies bacterianas aisladas de todos los sujetos

\begin{tabular}{|c|c|c|}
\hline $\begin{array}{c}\text { Especie } \\
\text { microbiana }\end{array}$ & $\begin{array}{c}\text { Frecuencia absoluta } \\
(\mathbf{n = 2 3})\end{array}$ & $\begin{array}{c}\text { \% del total } \\
\text { aislado }\end{array}$ \\
\hline$T d$ & 15 & 65.22 \\
\hline$T f$ & 15 & 65.22 \\
\hline$P g$ & 4 & 17.39 \\
\hline$A a$ & 3 & 13.04 \\
\hline
\end{tabular}

Se evaluó la detección de los patógenos según la severidad de periodontitis. Se observa que Treponema denticola fue aislada en el $40 \%$ de los sujetos con periodontitis leve, $66.6 \%$ con periodontitis moderada y en el $73.3 \%$ de los sujetos con diagnóstico de periodontitis severa. Tannerella forsythia se aisló en el $20 \%$ de los sujetos con periodontitis 
leve, $66.6 \%$ con periodontitis moderada y el $80 \%$ con periodontitis severa. Porphyromonas gingivalis y Aggregatibacter actinomycetemcomitans solo se apreciaron en sujetos con periodontitis leve y severa. Porphyromonas gingivalis en un $6.6 \%$ de los sujetos con periodontitis leve y $20 \%$ de periodontitis severa. Aggregatibacter actinomycetemcomitans en un $40 \%$ de periodontitis leve y $6.6 \%$ de periodontitis severa (Figura 5 ).

Un $80 \%$ de los sujetos con buen control glicémico presentó Treponema denticola, un 40\% Agreggatibacter actinomycetemcomitans y un $20 \%$ Tannerella forsythia. En el grupo de moderado control glicémico se aislaron Treponema denticola en un $81.8 \%$, Tannerella forsythia en un $72.7 \%$ y Porphyromonas gingivalis en un $27.2 \%$. Entre los sujetos con mal control glicémico se encontró un $85.7 \%$ de Tannerella forsythia, $28.5 \%$ de Treponema denticola, $14.2 \%$ de Porphyromonas gingivalis y $6.6 \%$ de Aggregatibacter actinomycetemcomitans (Figura 5).

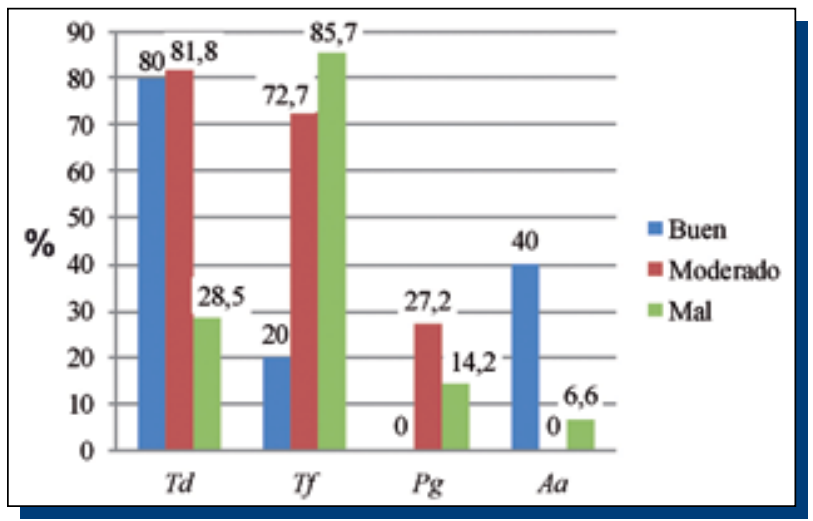

Figura 5. Distribución porcentual de las especies bacterianas en individuos con DM 2, según control glicémico Td: Treponema denticola; Tf: Tannerella forsythia; Pg: Porphyromonas gingivalis; Aa: Aggregatibacter actinomycemtencomitans.

\section{DISCUSIÓN}

La diabetes mellitus es un factor de riesgo de periodontitis y está asociado a mayores niveles de profundidad al sondaje, pérdida de nivel de inserción clínica y pérdida de piezas dentarias ${ }^{(11,15,16,17)}$. Estudios de asociación entre diabetes mellitus tipo 2 y enfermedad periodontal en USA han demostrado mayor incidencia, prevalencia, severidad y progresión de periodontitis al compararlos con sujetos no diabéticos ${ }^{(18-23)}$. En Brasil un estudio prospectivo reportó mayor severidad y progresión de periodontitis al comparar diabéticos tipo 2 y no diabéticos ${ }^{(24)}$. Varias investigaciones han relacionado un empeoramiento en el estado periodontal a medida que empeora el control glicémico de los pacientes diabéticos ${ }^{(16,24,25)}$. En nuestra investigación los grupos con moderado y mal control glicémico presentaron en promedio mayores valores de profundidad al sondaje, pérdida de inserción clínica y porcentaje de sitios con sangrado al sondaje, además de menor cantidad de piezas dentarias en comparación con el grupo de mejor control glicémico, mostrando una tendencia hacia un peor estatus periodontal a medida que el control glicémico empeora. Los datos más claros son los de porcentaje de sangrado al sondaje y la mediana de piezas dentarias en boca, que muestran mayores diferencias en sus valores en los grupos de moderado a mal control glicémico comparado con buen control glicémico lo que está en concordancia con estudios previos ${ }^{(26)}$. Posiblemente estas diferencias se harían más evidentes con un mayor tamaño de muestra.

Diferentes estudios han reportado diferencias en la flora microbiana subgingival entre países ${ }^{(27,28)}$. En Chile, se analizó el biofilm subgingival de 26 adultos chilenos sistémicamente sanos con periodontitis no tratada ${ }^{(27)}$, reportando una alta prevalencia de especies como Porphyromonas gingivalis (>90\%), Treponema denticola, Tannerella forsythia, Fusobacterium nucleatum, Campylobacter rectus, Peptostreptococcus micros entre otros y menor presencia de Actinomyces ssp. El análisis del PCR de nuestra investigación muestran una alta presencia de Treponema denticola y Tannerella forsythia, seguida de Porphyromonas gingivalis y Aggregatibacter actinomycetemcomitans (Tabla 5). Estos resultados son similares a los obtenidos por Makiura y cols $^{(29)}$, sin embargo contrastan con otros estudios que detectaron mayor presencia de Porphyromonas gingivalis que de Treponema denticola y Aggregatibacter actinomycetemcomitans ${ }^{(30-33)}$.

Al analizar la presencia de las bacterias estudiadas según severidad de la periodontitis, Treponema denticola, Tannerella forsythia y Porphyromonas gingivalis se presentaron en mayor porcentaje en los sujetos con periodontitis moderada y severa. La prevalencia de Porphyromonas gingivalis en nuestra investigación fue inferior a lo obtenido por otros autores, tanto en pacientes sistémicamente sanos $^{(34,35)}$, como en diabéticos ${ }^{(10,31-33)}$. Se ha observado que la proporción de especies como Porphyromonas gingivalis, Treponema denticola y Tannerella forsythia aumenta a medida que los valores de profundidad al sondaje son mayores ${ }^{(34)}$, lo que está en concordancia con lo observado en nuestro estudio en que el grupo de pacientes con periodontitis severa presentó mayor presencia de Porphyromonas gingivalis comparado con los grupos de periodontitis leve y moderada.

Al comparar la presencia de las bacterias estudiadas en relación al control glicémico se observó una tendencia a una mayor presencia de estas en los individuos con peor control metabólico. Estos resultados son similares a los obtenidos por Tervonen y cols $^{(36)}$, quien al investigar el biofilm subgingival de 107 diabéticos a través de inmunoensayo, reportó que la prevalencia de Aggregatibacter actinomycetemcomitans, Fusobacterium nucleatum, Eikenella corrodens, Porphyromonas gingivalis y Prevotella intermedia aumenta en la medida que disminuye el control metabólico. Investigaciones de Zambon y cols ${ }^{(32)}$ y Mandell y cols $^{(31)}$ reportaron las mismas conclusiones en diabéticos tipo 2 y 1 respectivamente. Estos resultados contrastan con los obtenidos por otros autores que si encontraron diferencias significativas entre la presencia de determinados patógenos periodontales y el control glicémico ${ }^{(24,29)}$. Una explicación a estas importantes diferencias en los resultados estaría dada por los sujetos diabéticos que participaron en nuestra investigación, todos los cuáles se encuentran en tratamiento y control permanente por un diabetólogo especialista. La Asociación Americana de Diabetes y el Ministerio de Salud de Chile consideran como mal control glicémico valores de hemolobina glicosilada sobre $7 \%$ e inestabilidad metabólica sobre $9 \%$, basado en estudios que demuestran la asociación de complicaciones crónicas con estos valores (DCCT y UKPDS). Gran parte de las investigaciones realizadas previamente en pacientes diabéticos, consideran como un control glicémico malo niveles de hemoglobina glicosilada mayores a $9-10 \%$, superiores a la definición de casos de nuestro estudio. Posiblemente incluir diabéticos con mayores niveles de hemoglobina glicosilada resultaría en cambios significativos en el estatus periodontal y quizás en la flora microbiana. Otra situación que limita el análisis es el escaso tamaño muestral de nuestra investigación. Si bien éste es limitado se puede apreciar una tendencia en que la severidad de la periodontitis y el mal control metabólico influenciarían la composición de la flora microbiana subgingival.

En nuestra investigación se observó una mayor severidad de periodontitis, además de menor cantidad de piezas dentarias en el grupo de pacientes DM2 con peor control glicémico en comparación con el grupo de mejor control glicémico. Fue posible aislar especies de Treponema denticola, Tannerella forsythia, Porphyromonas gingivalis y Aggregatibacter actinomycetemcomitans del biofilm sibgingival de pacientes con diabetes mellitus 2. El grupo con periodontitis severa presentó mayor presencia de $T d, T f, P g$ y $A a$, comparado con el grupo de periodontitis leve. Los pacientes diabéticos tipo 2 con moderado y mal control glicémico presentaron mayor presencia de $T d, T f, P g$ y $A a$, comparado con los grupos con mejores niveles de control glicémico.

Nuestra investigación tiene un carácter descriptivo y sus resultados se circunscriben a una muestra pequeña de enfermos diabéticos (23 pacientes). Nuestro grupo de investigación está realizando nuevos estudios en el ámbito de la relación diabetes-periodontitis con un tamaño de muestra mayor que permitirá realizar comparaciones entre pacientes de acuerdo a su estatus periodontal y nivel de control glicémico.

\section{AGRADECIMIENTOS}

A Universidad de los Andes por el apoyo financiero, a través del proyecto FAI ODO, que permitió realizar esta investigación.

\section{CONFLICTOS DE INTERÉS}

Los autores declaran no presentar conflictos de interés de ningún tipo. 


\section{REFERENCIAS BIBLIOGRÁFICAS}

1. Mealey BL, Ocampo GL. Diabetes mellitus. Periodontol 2000, 2007; 44: $127-153$

2. American Diabetes Association. Diagnosis and clasificación of diabetes mellitus. Position statement. Diabetes Care, 2005; 29(1): 37-42.

3. Gobierno de Chile, Ministerio de Salud. Resultados Encuesta de Salud, Chile 2009. Disponible http://epi.minsal.

4. Bacic M, Plancak D, Granic M. CPITN assessment of periodontal status in diabetic patients. J Periodontol, 1988; 59: 816-822.

5. Tervonen T, Oliver R. Long-term control of diabetes mellitus and periodontitis. J Clin Periodontol, 1993; 20: 431-435.

6. Mealey BL, Oates TW. Diabetes mellitus and periodontal diseases. $J$ Periodontol, 2006: 1289-1303.

7. Taylor GW, Burt BA, Becker MP, Genco RJ, Shlossman M, Knowler WC et al. Severe periodontitis and risk for poor glycemic control in patients with non-insulin-dependent diabetes mellitus. J Periodontol, 1996; 67: 1085-1093.

8. Thorstensson $\mathrm{H}$, Kuylenstierna $\mathrm{J}$, Hugoson A. Medical status and complications in relation to periodontal disease experience in insulindependent diabetics. J Clin Periodontol, 1996; 23: 194-202.

9. Löe H. Periodontal disease. The sixth complication of diabetes mellitus. Diabetes Care, 1993; 16: 329-334.

10. Ebersole JL, Holt SC, Hansard R, Novak MJ. Microbiologic and immunologic characteristics of periodontal disease in Hispanic americans with type 2 diabetes. J Periodontol, 2008; 79(4): 637-646.

11. Papapanou PN. World workshop in clinical periodontics. Periodontal diseases: Epidemiology. Ann Periodontol, 1996; 1: 1-36.

12. Ciantar M, Gilthorpe MS, Hurel SJ, Newman HN, Wilson M, Spratt DA. Capnocytophaga spp. in periodontitis patients manifesting diabetes mellitus. J Periodontol, 2005; 76(2): 194-203.

13. American Diabetes Association. Diagnosis and classification of diabetes mellitus (Position Statement). Diabetes Care, 2010; 33 (Suppl. 1): 62-69.

14. Page RC, Eke PI. Case definitions for use in population-based surveillance of periodontitis. J Periodontol, 2007; 78(7): 1387-1399.

15. Taylor GW. Bidirectional interrelationships between diabetes and periodontal diseases: An epidemiologic perspective. Ann Periodontol, 2001; 6: 99-112.

16. Taylor GW, Borgnakke WS. Periodontal disease: Associations with diabetes, glicemia control and complications. Oral Diseases, 2008; 14: 191-203.

17. Mealey BL. Periodontal disease and diabetes: A two-way street. J Am Dent Assoc, 2006; 137: 26-31.

18. Emrich LJ, Shlossman M, Genco RJ. Periodontal disease in noninsulin dependent diabetes mellitus. J Periodontol, 1991; 62: 123-3.

19. Shlossman M, Knowler WC, Pettitt DJ, Genco RJ. Type 2 diabetes mellitus and periodontal disease. J Am Dent Assoc, 1990; 121: 532-536. 20. Nelson RG, Shlossman M, Budding LM, Pettitt DJ, Saad MF, Genco RJ, Knowler WC. Periodontal disease and NIDDM in Pima Indians. Diabetes Care, 1990; 13(8): 836-840.

21. Taylor GW, Burt BA, Becker MP, Genco RJ, Shlossman M, Knowler WC, Pettitt DJ. Non-insulin dependent diabetes mellitus and alveolar bone loss progression over 2 years. J Periodontol, 1998; 69(1): 76-83.
22. Rodenburg JP, vanWinkelhoff AJ, Winkel EG, Goene RJ, Abbas F, de Graff J. Occurrence of Bacteroides gingivalis, Bacteroides intermedius and Actinobacillus actinomycetem-comitans in severe periodontitis in relation to age and treatment history. J Clin Periodontol, 1990; 17: 392-399.

23. Grossi SG, Zambon JJ, Ho AW. Assessment of risk for periodontal disease. I. Risk indicators for attachment loss. J Periodontol, 1994; 65: 260-267.

24. Novaes AB Jr., Gutiérrez FG, Novaes AB. Periodontal disease progression in Type II non-insulin-dependent diabetes mellitus patients (NIDDM). Part I-Probing pocket depth and clinical attachment. Braz Dent, 1996; 7: 65-73.

25. Taylor GW, Burt BA, Becker Mp, Genco RJ. Glycemic control and alveolar bone loss progression in type 2 diabetes. Ann Periodontol, 1998; 3: 30-39.

26. Kaur G, Holtfreter B, Rathmann W, Schwahn C, Wallaschofski H, Schipf S, Nauck M, Kocher T. Association between type 1 and type 2 diabetes with periodontal disease and tooth loss. J Clin Periodontol, 2009; 36: 765-774.

27. Haffajee AD, Bogren A, Hasturk H, Feres M, Lopez NJ, Socransky SS. Subgingival microbiota of chronic periodontitis subjects from different geographic locations. J Clin Periodontol, 2004; 31: 996-1002.

28. Herrera D, Contreras A, Gamonal J, Oteo A, Jaramillo A, Silva N, Sanz M, Botero JE, León R. Subgingival microbial profiles in chronic periodontitis patients from Chile, Colombia and Spain. J Clin Periodontol, 2008; 35: 106-113.

29. Makiura N, Ojima M, Kou Y. Relationship of Porphyromonas gingivalis with glycemic level in patients with type 2 diabetes following periodontal treatment. Oral Microbiology Inmunology, 2008; 23: 348-351.

30. Sastrowijoto $H$, Hillemans $P$, van Steenbergen $T$, Abraham-Inpijn L, Graaff J. Periodontal condition and microbiology of healthy and diseased periodontal pockets in type 1 diabetes mellitus patients. J Clin Periodontol, 1989; 16(5): 316-322.

31. Mandell RL, Dirienzo J, Kent R et al. Microbiology of healthy and diseased periodontal sites in poorly controlled insulin dependent diabetics. J Periodontol, 1992; 63: 274-279.

32. Zambon JJ, Reynolds H, Fisher JG, Shlossman M, Dunford R, Genco RJ. Microbiological and immunological studies of adult periodontitis in patients with non insulin-dependent diabetes mellitus. J Periodontol, 1988; 59(1): 23-31.

33. Yuan K, Chang C-J, Hsu P-C, Sun HS, Tseng C-C, Wang J-R. Detection of putative periodontal pathogens in non-insulin-dependent diabetes mellitus and non-diabetes mellitus by polymerase chain reaction. J Periodont Res, 2001; 36: 18-24.

34. Socransky SS, Haffajee AD, Cugini MA, Smith C, Kent RL Jr. Microbial complexes in subgingival plaque. J Clin Periodontol, 1998 Feb; 25(2): 134-144.

35. Socransky S, Haffajje A. Periodontal microbial ecology. Periodontol 2000, 2005; 38: 135-187.

36. Tervonen T, Oliver R, Wolff L, Bereute $r$ J, Anderson L, Aeppli D. Prevalence of periodontal pathogens with varying metabolic control of diabetes mellitus. J Clin Periodontol, 1994; 21(6): 375-379. 